\title{
Effect of mucosal suture on the healing of mucosal defect in laparoscopic intragastric surgery
}

\author{
Takeyoshi Yumiba ${ }^{1}$, Toshinori ito ${ }^{1}$, Hirofumi Ikushima ${ }^{1}$, Eiji Taniguchi ${ }^{2}$, Yoshifumi Inoue ${ }^{1}$, \\ Toshirou Nishida ${ }^{1}$, Toru Kitagawa ${ }^{1}$, Kazuhiro Nishikawa ${ }^{1}$, Shuichi Ohashi ${ }^{2}$, and Hikaru Matsuda ${ }^{1}$ \\ ${ }^{1}$ Department of Surgery, Osaka University Graduate School of Medicine, Osaka, Japan \\ ${ }^{2}$ Department of Endoscopic Surgery, Osaka University Graduate School of Medicine, Osaka, Japan
}

\begin{abstract}
Background. The efficacy of mucosal suturing for the healing of a mucosal defect in laparoscopic intragastric surgery (LIGS) for gastric lesions is not yet known.

Methods. We prospectively studied ten patients who underwent mucosal resection by LIGS for gastric tumors: four patients with early gastric cancer and six with gastric adenoma. Patients were randomly divided into two groups: group I $(n=$ 5); patients who underwent mucosal resection by LIGS with mucosal defect suturing and group II $(n=5)$; patients who underwent mucosal resection by LIGS without mucosal defect suturing. We performed endoscopy on day 10, and 1 month, 2 months, and 3 months after the operation to observe the healing process of the mucosal defect. The ulcer stage by endoscopy was classified as active, healing, or scarring according to the classification of Sakita and colleagues. Patients were given an $\mathrm{H}_{2}$-blocker daily until the mucosal defect improved to the scarring stage.

Results. There were no significant differences in sex, age, tumor location, size of mucosal resection, or the incidence of Helicobacter pylori infection between groups I and II. The ulcer stages in group I were significantly lower than those in group II on day 10, and 1 month, 2 months, and 3 months postoperation. The medication cost (H2-blocker) in group I was significantly lower than that in group II.

Conclusion. Mucosal defect suturing after mucosal resection by LIGS promotes more rapid healing of mucosal defects and reduces drug costs for patients.
\end{abstract}

Key words Laparoscopic intragastric surgery · Gastric cancer $\cdot$ Mucosal suture $\cdot$ Ulcer $\cdot$ Healing

Offprint requests to: T. Yumiba, Department of Surgery, Osaka Kosei-Nenkin Hospital, 4-2-78 Fukushima, Fukushima-ku, Osaka 553-0003, Japan

Received: September 5, 2002 / Accepted: January 9, 2003

\section{Introduction}

Recently, laparoscopic intragastric surgery (LIGS) has been employed for early gastric cancer and gastric leiomyoma [1,2]. It has not yet been determined whether mucosal defect suturing improves mucosal wound healing after mucosal resection by LIGS. The efficacy of suturing the mucosal defect for wound healing is not yet known.

The purpose of this study was to determine the influence of mucosal suturing on the healing process of a mucosal defect in laparoscopic intragastric mucosal resection for gastric lesions.

\section{Patients and methods}

We prospectively studied ten patients who underwent mucosal resection by LIGS for gastric tumors: four patients with early gastric cancer and six with gastric adenoma. Mucosal resection by LIGS was performed according to the method of Ohashi [1]. Patients were randomly divided into two groups: group I $(n=5)$; patients who underwent mucosal resection by LIGS with mucosal defect suturing and group II $(n=5)$; patients who underwent mucosal resection by LIGS without mucosal defect suturing. There were two patients with gastric cancer and three with gastric adenoma in each of groups I and II. The mucosal defect was closed with a continuous suturing of 4-0 Vicryl (Ethicon, Cincinnati, OH, USA). We performed endoscopy on day 10, and 1 month, 2 months, and 3 months after the operation to observe the healing process of the mucosal defect. The mucosal defect became an ulcer. The ulcer stage by endoscopy was classified as active, healing, or scarring, according to the classification of Sakita and colleagues [3] and Miyake and colleagues [4]. Briefly, these stages are defined as follows: active stages (A1, A2), the surrounding mucosa is edematous 
and regenerating epithelium is not seen endoscopically; healing stages $(\mathrm{H} 1, \mathrm{H} 2)$, the white coating becomes thin, and the regenerating epithelium extends into the ulcer; and scarring stages (S1, S2), the regenerating epithelium completely covers the bottom of the ulcer. Stage A1 corresponds to the acute phase of active ulceration, with a thick white coating over the lesion, edema surrounding the lesion, or hemorrhage; and stage A2 corresponds to the subacute phase of active ulceration, with initial healing and white coating within the lesion. Stage $\mathrm{H} 1$ corresponds to the healing stage, with obvious marginal regeneration of the epithelium, shallow ulcer, and smooth round marginal white coating, and stage $\mathrm{H} 2$ corresponds to the late healing stage with obviously decreasing size of ulcer and thin white coating. Stage S1 corresponds to the phase when the ulcer crater has just disappeared and stage S2 corresponds to the phase when the mature, regenerated mucosa presents a cobblestone-like appearance. Patients were given an $\mathrm{H}_{2}$-blocker daily until the mucosal defect improved to the scarring stage.

In order to compare ulcer stages between groups I and II, ulcer stages were replaced by scores: A1, 6; A2, 5; H1, 4; H2, 3; S1, 2; and S2, 1. The Mann-Whitney $U$-test was used to compare data (ulcer stages: score) between groups I and II. A value of $P<0.05$ was considered significant.

\section{Results}

There were no significant differences in sex, age, tumor location, size of mucosal resection, or the incidence of Helicobacter pylori infection between groups I and II (Table 1). The maximum diameters of the gastric cancers in group I were 10 and $12 \mathrm{~mm}$, and those of the gastric adenomas were 8,12 , and $30 \mathrm{~mm}$. In group II, the maximum diameters of the gastric cancers were 6 and $13 \mathrm{~mm}$, and those of the gastric adenomas were 8,14 , and $15 \mathrm{~mm}$. There were no significant differences in tumor sizes between groups I and II.

Chronological endoscopic findings in representative patients from groups I and II are shown in Fig. 1. Mu-

Table 1. Clinical characteristics of the two groups

\begin{tabular}{lll}
\hline & \multicolumn{1}{c}{ Group I } & \multicolumn{1}{c}{ Group II } \\
\hline Male:Female & $3: 2$ & $2: 3$ \\
Age; years (mean) & $57-71(63.8)$ & $56-66(61.3)$ \\
Location & $\mathrm{U}, 2 ; \mathrm{M}, 2 ; \mathrm{L}, 1$ & $\mathrm{U}, 2 ; \mathrm{M}, 3$ \\
Resected size & $31.8 \pm 2.0 \mathrm{~mm}$ & $29.0 \pm 8.0 \mathrm{~mm}$ \\
Helicobacfer pylori $(+)$ & $3 / 5$ & $2 / 5$ \\
\hline
\end{tabular}

Group I $(n=5)$, patients with suturing of mucosal defect after laparoscopic intragastric surgery (LIGS); group II $(n=5)$, patients without suturing of mucosal defect after LIGS; U, upper stomach; $\mathrm{M}$, middle stomach; L, lower stomach

${ }^{a}$ Data values are expressed as means $\pm \mathrm{SD}$ cosal defects (the resected area) healed in a manner similar to that of a peptic gastric ulcer, i.e., they healed in a step-by-step fashion, in both groups I and II.

Endoscopy at 10 days revealed that in group I, one patient had an A2 stage ulcer and four had H1 stage ulcers, while in group II, two patients had A1 stage ulcers and three had A2 stage ulcers (Fig. 2). The ulcer stages in group I were significantly lower $(P=0.011)$ than those in group II.

Endoscopy at 1 month revealed that all patients in group I had $\mathrm{H} 2$ stage ulcers, while in group II, one patient had an A2 stage ulcer and four had H1 stage ulcers (Fig. 2). The ulcer stages in group I were significantly lower $(P=0.004)$ than those in group II.

Endoscopy at 2 months revealed that one patient in group I, had an $\mathrm{H} 2$ stage ulcer and four had S1 stage ulcers, while all patients in group II had $\mathrm{H} 2$ stage ulcers (Fig. 2). The ulcer stages in group I were significantly lower $(P=0.014)$ than those in group II.

Endoscopy at 3 months revealed that all patients in group I had S2 stage ulcers, while all patients in group II had S1 stage ulcers (Fig. 2). The ulcer stages in group I were significantly lower $(P=0.003)$ than those in group II.

Patients who had taken $\mathrm{H}_{2}$-blockers daily until the mucosal defect reached the scarring stage had medication costs of 5000 yen a month (30 days). The overall cost of $\mathrm{H}_{2}$-blockers in group I $(11000 \pm 2000$ yen $)$ was significantly lower than that in group II (15000 \pm 0 yen).

\section{Discussion}

The resected mucosal area was left untreated as a defect after mucosal resection by LIGS in the original method [1]. However, how a mucosal defect heals after mucosal resection by LIGS has not been determined. Peptic gastric ulcer heals in a step-by-step fashion; active stage (A1, A2), healing stage ( $\mathrm{H} 1, \mathrm{H} 2)$, and scarring stage (S1, S2) [3]. The present study showed that the mucosal defects after mucosal resection healed in a manner similar to that of a peptic gastric ulcer, in both group I and group II. Hashimoto and Adachi [5] demonstrated that endoscopic mucosal resection (EMR)-induced ulcers might be one of the best human acute gastric ulcer models. The defect after mucosal resection by LIGS is thought to be essentially the same as that in EMR-induced ulcers. So, we assumed that the biological behaviour of the defect after mucosal resection might be the same as that following a peptic ulcer.

Recently, it has not been particularly difficult to suture mucosal defects by a laparoscopic procedure, because suturing instruments and techniques have ad- 


\section{0th day}

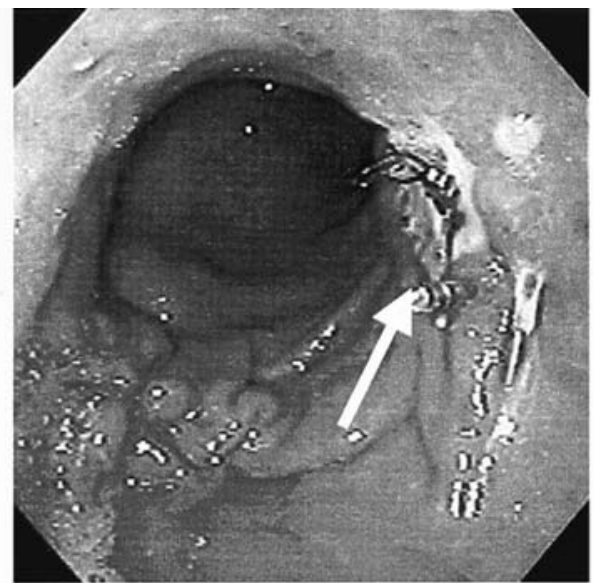

\section{2nd month}

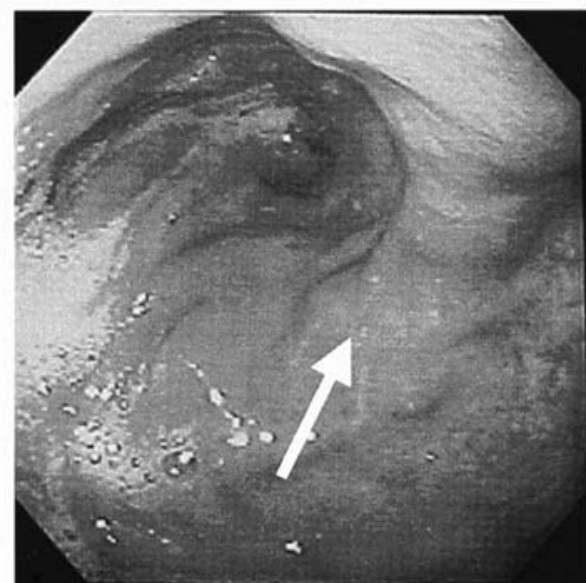

\section{0th day}

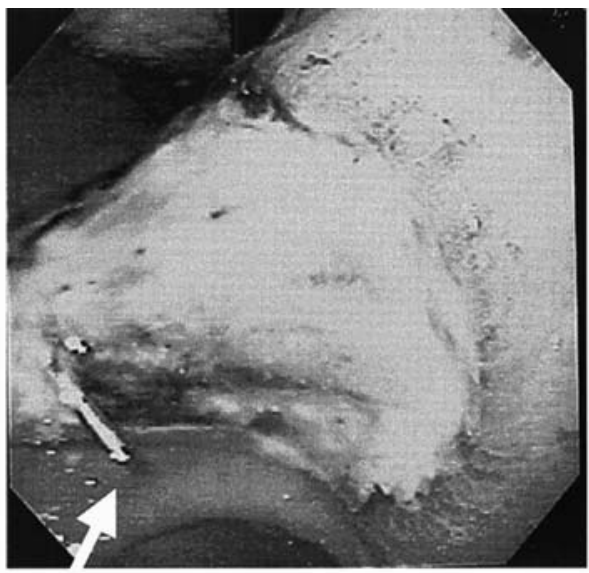

2nd month

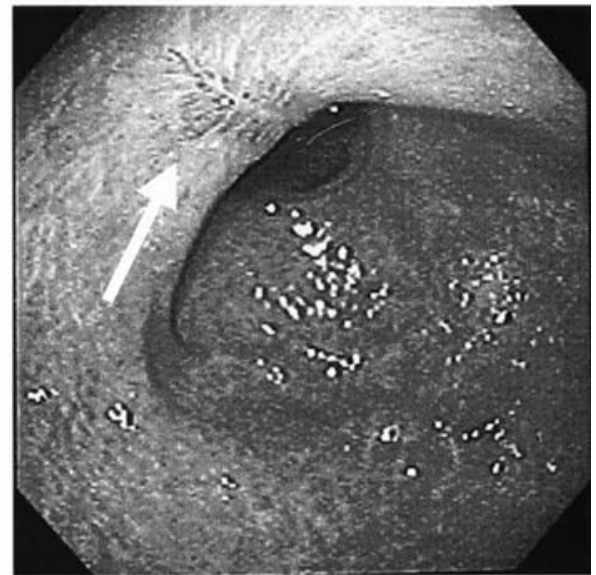

Fig. 1. Chronological endoscopic findings in representative patients from groups I and II. Arrows show mucosal defects (ulcers) in each endoscopic examination. Left, the ulcer stages in group I (with mucosal defect suturing) were H1 on the tenth day and $\mathrm{S} 1$ at 2 months. Right, the ulcer stages in group II (without mucosal defect suturing) were A2 on the tenth day and H2 at 2 months

vanced. Armstrong et al. [6] demonstrated that an ulcer size of more than $15 \mathrm{~mm}$ in diameter and multiple ulcers were factors associated with decreasing healing rates or slow healing in peptic ulcers. Ulcer size is believed to play an important role in the healing of gastric ulcers. It has not yet been determined whether mucosal defect suturing is better for healing mucosal defects after mucosal resection by LIGS. The present study showed the ulcer healing in group I to be significantly faster than that in group II at every examination (10 days and 1, 2, and 3 months after the operation). Mucosal defect suturing after mucosal resection by LIGS was thought to promote rapid healing of the mucosal defect.

Whether the LIGS patient should be given an $\mathrm{H}_{2}-$ blocker or a proton pump inhibitor to promote rapid healing of a mucosal defect has yet to be determined. The patient who undergoes EMR for gastric lesions is usually given an $\mathrm{H}_{2}$-blocker or a proton pump inhibitor
[5]. We thought that $\mathrm{H}_{2}$-blockers were needed after mucosal resection by LIGS, similarly to the need after EMR. We gave $\mathrm{H}_{2}$-blockers to the patients who underwent mucosal resection by LIGS until the mucosal defect reached the scarring stage. Four of the five patients $(80 \%)$ in group I, but none of the group II patients, were able to discontinue the $\mathrm{H}_{2}$-blockers at 2 months after the operation. Finally, all group II patients and one in group I stopped taking $\mathrm{H}_{2}$-blockers at 3 months after the operation. Accordingly, mucosal defect suturing is thought to provide an economic benefit in terms of drug costs. However, further study would be needed to determine whether $\mathrm{H}_{2}$-blockers are really needed after mucosal resection by LIGS.

In conclusion, mucosal defect suturing after mucosal resection by LIGS promotes more rapid healing of the mucosal defect and reduces drug costs for patients. 


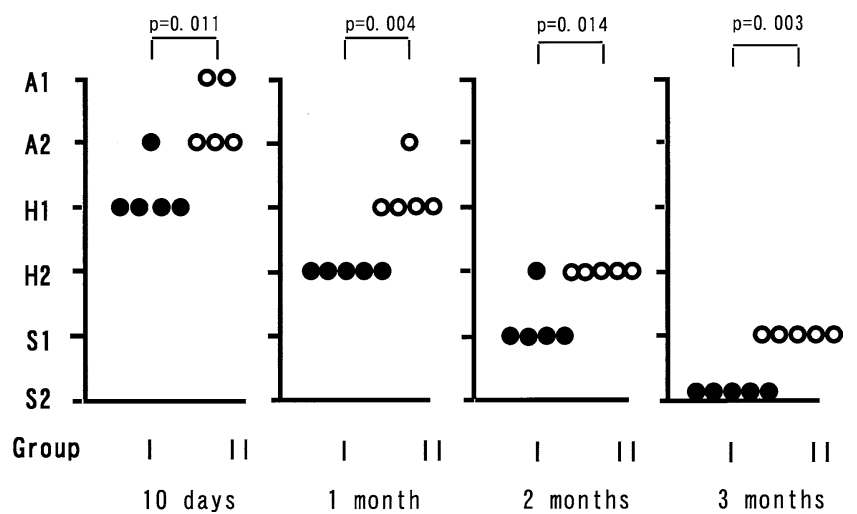

Fig. 2. Chronological healing stage of the mucosal defects in groups I and II. The mucosal defects were assigned to one of the six stages $(A 1, A 2, H 1, H 2, S 1$, and $S 2)$ according to Sakita's endoscopic classification of gastric ulcer. Group I $(n=5)$, patients who underwent mucosal resection by laparoscopic intragastric surgery (LIGS) with mucosal defect suturing; group II $(n=5)$, patients who underwent mucosal resection by LIGS without mucosal defect suturing. The ulcer stages in group I were significantly lower $(P=0.011)$ than those in group II at the 10-day endoscopic examination. The ulcer stages in group I were significantly lower $(P=0.004)$ than those in group II at 1 month. The ulcer stages in group I were significantly lower $(P=0.014)$ than those in group II at 2 months. The ulcer stages in group I were significantly lower $(P=0.003)$ than those in group II at 3 months

\section{References}

1. Ohashi S. Laparoscopic intraluminal (intragastric) surgery for early gastric cancer. A new concept in laparoscopic surgery. Surg Endosc 1995;9:169-71.

2. Taniguchi E, Kamiike W, Yamanishi H, Ito T, Nezu R, Nishida T, et al. Laparoscopic intragastric surgery for gastric leiomyoma. Surg Endosc 1997;11:287-9.

3. Sakita T, Oguro Y, Takasu S, Fukutomi H, Miwa T, Yoshimori M. Observations on the healing of ulcerations in early gastric cancer. The life cycle of the malignant ulcer. Gastroenterology 1971;60: 835-9.

4. Miyake T, Suzaki T, Oishi M. Correlation of gastric ulcer healing features by endoscopy, stereoscopic microscopy, and histology, and a reclassification of the epithelial regenerative process. Dig Dis Sci 1980;25:8-14.

5. Hashimoto T, Adachi K. Changes in gastric mucosal blood flow during healing of EMR-induced ulcer - comparison with peptic ulcer. Dig Endosc 1997;9:127-31.

6. Armstrong D, Arnold R, Classen M, Fischer M, Goebell H, Blum AL. Prospective multicentre study of risk factors associated with delayed healing of recurrent duodenal ulcers (RUDER). RUDER Study Group. Gut 1993;34:1319-26. 